\title{
Contingent aftereffects: Lateral interactions between color and motion
}

\author{
LINDSAY T. SHARPE \\ Neurologische Universitätsklinik, Freiburg im Breisgau, Germany \\ JOHN P. HARRIS \\ University of Bristol, Bristol, England \\ and \\ CLEMENS C. FACH and DORIS I. BRAUN \\ Neurologische Universitätsklinik, Freiburg im Breisgau, Germany
}

\begin{abstract}
A randomly dotted yellow disk was rotated at a speed of $5 \mathrm{rpm}$, alternating in direction every $10 \mathrm{sec}$. Its change in direction of rotation was paired with a change in surround color, which was either red or green. After $15 \mathrm{~min}$ of exposure, observers reported vivid motion aftereffects contingent on the color of both the stationary disk and the surround, even though during adaptation only motion or color was associated with either alone. In further experiments, it was established that a change in color (or direction of motion) of the disk could be associated with a change in direction of motion (or color) of the surround. Such lateral effects were found even when a wide $\left(5^{\circ}\right)$ annulus was introduced between the disk and the surround during adaptation and testing. Furthermore, the aftereffects generalized to the annulus, which was not associated with either color or motion during adaptation. However, when the disk alone was adapted to color and motion, no generalization to the surround was found (and vice versa), suggesting that the effects are not produced by adaptation of large receptive fields or by scatter of light within the eye. The results appear to conflict with the ideas that contingent aftereffects are confined to the adapted area of the retina and that they are built up by links between single-duty neurones, and with an extreme view of the segregation of color and motion early in human vision.
\end{abstract}

When the elements of a moving display are segregated from the background by color rather than luminance, motion perception is degraded. If a random-dot kinematogram is made equiluminant, the perception of coherent motion is lost (Ramachandran \& Gregory, 1978; Troscianko, 1987). The apparent velocity of equiluminant gratings, dots, or bars is reduced, compared with that of low-luminancecontrast versions of the same stimuli (Cavanagh, Tyler, \& Favreau, 1984; Troscianko \& Fahle, 1988). One interpretation of such findings is that motion-detecting mechanisms receive most of their input from luminance, rather than from chromatic channels, and that color and motion information are in some sense segregated early in visual

A summary of part of this work was presented at the 11th European Conference on Visual Perception, Bristol, England, 31st August to 3rd September 1988 and published in Perception, 17 (1988), 406. The experiments were supported by the Deutsche Forschungsgemeinschaft (SFB 325, B4), Bonn-Bad Godesberg. We thank Lothar A. Spillmann and two anonymous referees for improving the manuscript. John $P$. Harris's present address is: Department of Psychology, University of Reading, Earlie Gate, Whiteknights, Reading RG6 2AL, England. Doris I. Braun's present address is: Department of Neurology, Santa Clara Valley Medical Center, 751 South Basom Avenue, San Jose, CA 95128. Requests for reprints should be sent to Lindsay T. Sharpe, Neurologische Universitätsklinik, Hansastrasse 9, D-7800, Freiburg im Breisgau, Germany. processing (Carney, Shadlen, \& Switkes, 1987). Support for this idea can be drawn from anatomical evidence for separate projection areas for motion and color information (DeYoe \& Van Essen, 1988; Shapley, 1990; Van Essen, 1986; Van Essen \& Maunsell, 1983; Zeki, 1978).

However, an older, if less fashionable, class of observations suggests that hypotheses about a general segregation of color and motion should be treated with caution. In particular, motion aftereffects contingent on color (Favreau, Emerson, \& Corballis, 1972; Mayhew \& Anstis, 1972) and their complementary, colored aftereffects contingent on direction of motion (Hepler, 1968) suggest that strong perceptual links between color and motion exist or can be built up. These perceptual aftereffects are usually assumed to reflect adaptive changes in early visual processing (see, e.g., Anstis, 1975), and they are believed to be confined to the adapted area of the retina (Stromeyer, 1978).

In principle, there is no problem in reconciling the two kinds of effects: With equiluminant phenomena, such as random-dot kinematograms, the moving elements are defined only by color contrast, whereas with contingent motion aftereffects (CMAEs), the moving elements are defined by luminance contrast but their direction of motion is linked to a particular color. Thus CMAEs and equiluminant motion stimuli may tap the association between color and motion at different levels of visual analysis, and 
this may yield different answers about the existence and nature of such an association.

Although the neural site of color-CMAEs is uncertain, the demonstrations of Murch (1976) and Potts and Harris (1979) that, after dichoptic adaptation, color-CMAEs can be obtained only when the eye that has seen color during adaptation also sees it during testing, suggest a monocular component, and so place the effects early in human visual processing. The nature of the processes underlying CMAEs is controversial (for a review, see Skowbo, Timney, Gentry, \& Morant, 1975): Some authors (e.g., McCollough, 1965) have suggested that color-CMAEs reflect adaptation in double-duty units sensitive to both color and motion (presumably at the cortical level); others (e.g., Mayhew \& Anstis, 1972), that they result from links between single duty neurones (residing at retinal, lateral geniculate and/or cortical levels); and yet others (e.g., Wolfe, 1985), that they involve neuronal fatigue (depletion of neurotransmitter) in association with structural changes (strengthening or creation of synapses).

In the present paper, we investigate the adaptive links between color and motion processing mechanisms for different areas of the visual field, exploiting the finding of Potts and Harris (1975) that the perceived direction of motion of an achromatic stimulus can be made contingent on the color of the stationary surrounding area. Our results suggest that color information in one part of the visual field can influence motion processing in another, even when the two areas are separated by almost $5^{\circ}$ of visual angle. This linking of information is at least partly symmetrical-color processes can have access to motion processes, and vice versa-and seems to depend on lateral interactions between quite widely separated regions of the visual field. These effects may be related to remote surround effects on the properties of receptive fields (see, e.g., Valberg, Lee, Creutzfeldt, \& Tigwell, 1985). Our data show further that color-CMAEs can be obtained, on stationary colored test patterns, from retinal areas that have not adapted to either moving or colored patterns, when these lie between separated areas that are themselves adapted to color and motion. When, however, only one area of the retina is adapted to color and motion, there is no comparable spread of adaptation, suggesting that the spatial transfer of contingent adaptation found in the other experiments does not result from the adaptation of very large receptive fields, or from scattered light within the eye. In the Discussion, we argue that such effects cast doubt on the dogma of the retinal area specificity of CMAEs, and on the idea that CMAEs result from the build-up of links between single-duty neurones.

\section{GENERAL METHOD}

\section{Subjects}

The four authors acted as observers in all experiments. In some experiments, their results were confirmed by 8 naive subjects. No important differences were found between the results from the experienced subjects and the results from the naive subjects. The subjects were run individually or in pairs. They all had normal color vision and viewed the displays binocularly.

\section{Apparatus}

The stimulus display was viewed at a distance of $120 \mathrm{~cm}$. It consisted of an inner disk, $20 \mathrm{~cm}\left(9.5^{\circ}\right.$ of visual angle $)$ in diameter, and an annular surround, whose outer diameter was $50 \mathrm{~cm}\left(23.5^{\circ}\right)$. The inner diameter of the surround depended on the width of a dark annulus, whose inner diameter was always $20 \mathrm{~cm}$ and was contiguous with the outer edge of the disk. We shall refer to these different parts of the display as the disk, the surround, and the annulus. They were presented against a black masking screen, which subtended the observer's entire visual field of view when the observer was fixating the center of the stimulus display. The room was darkened to exclude all ambient light.

The disk and surround were formed of white cardboard. In some conditions, they were covered randomly with superimposed black dots, which were $7 \mathrm{~mm}\left(0.3^{\circ}\right.$ of visual angle) in diameter. When only unidirectional movement of the display was required (i.e., the disk or surround alone), the moving component of the adapting display was mounted on the rotating axle of a reversible synchronous motor, and any stationary component was held fixed in place. When simultaneous bidirectional rotation was required (i.e., disk and surround rotating in opposite directions), a pulley/cog-wheel system was attached to the motor axle to reverse the direction of rotation of the surround relative to the disk.

The disks and surrounds were front-illuminated by projectors (Leitz Prado Universal), equipped with tungsten filament lamps (Philips, $25 \mathrm{~V}, 250 \mathrm{~W}$ ). Various combinations of red (Kodak Wratten No. 25; dominant wavelength in CIE Illuminant $A=617 \mathrm{~nm}$ ), green (Kodak Wratten No. 59; $532 \mathrm{~nm}$ ), and yellow (Kodak Wratten No. 12; $586 \mathrm{~nm}$ ) filters were inserted in the projectors and used to color the disk and surround. In some experiments, a red (Kodak Wratten CCO5R) or a green (CCO5G) color-correcting filter was added to the yellow filter to cancel, respectively, the greenish tinge induced in the disk by the simultaneous color contrast from the red surround and the pinkish tinge induced by the green surround. Doing so introduced a wavelength difference (as opposed to a perceived color difference) of about $3 \mathrm{~nm}$ (586 vs. $589 \mathrm{~nm}$ ). To prevent unwanted light from falling on inappropriate parts of the display, disks or annuli were cut from opaque sticky labels with cork borers and mounted on the slides holding the filters.

The luminances of the bright parts of the disk and annulus were measured with a Minolta luminance meter. To prevent the buildup of MAEs contingent on brightness differences, rather than color differences, the luminances were set equal for different colors at $140 \mathrm{~cd} / \mathrm{m}^{2}$, by means of Schott neutral density filters inserted into the projector beams. An adjustable tachistoscope timing circuit was used to switch shutters (attached to electromagnetic servomotors) in and out of the projector beams, in synchrony with the reversal of rotation of the disk and/or surround.

\section{Procedure}

Before the adaptation period, the observers were tested with the stationary, alternating adaptation patterns and asked to report whether they perceived any change in the patterns. None reported any change other than in color. The observers were also asked to confirm by eye that the different colors were roughly matched for equal brightness.

The adaptation period lasted $15 \mathrm{~min}$. During the first $10 \mathrm{~min}$, the disk and/or surrounds were rotated at a speed of $5 \mathrm{rpm}$, alternating in direction every $10 \mathrm{sec}$. In synchrony with the motion reversal of the disk and/or surround, the color of the disk and/or surround changed (for details, see the Method sections for the individual experiments). The observers were instructed to fixate the center of the disk during adaptation. During the last 5 min of the adaptation period, the rate of alternation of the stimulus fields was increased to once every $5 \mathrm{sec}$. This control prevented the build-up of a large simple MAE on the last exposure (cf. Mayhew \& Anstis, 1972).

At the end of the adaptation period, there was a 2-min dark period before the beginning of the test period. To test for MAEs, the ob- 
servers were presented with the stationary disk and surround pattern, the color of which changed every $10 \mathrm{sec}$. The duration of the CMAEs was measured with a stopwatch, usually over 4 or 5 consecutive presentations of the appropriate test field. Observers were asked to report any apparent movement of the dots of the disk, the annulus (when it was present), and the surround and to state that movement's direction. The observers were also tested with various other combinations of stationary colored disks and surrounds to determine the generality and transferability of the CMAEs.

To prevent carry-over effects from biasing the results of subsequent experiments during the course of the experimental series, several precautions were taken. The particular colors associated with the two directions of motion during any adaptation sequence were randomly varied between experimental sessions. In some cases, if the adaptation conditions were the same in successive experiments, opposite pairings of color and motion were used in the subsequent experiment. Individual experiments were usually conducted on different days.

\section{EXPERIMENT 1}

We had three aims in the first experiment. First, we wanted to confirm whether the surround contingent effects reported by Potts and Harris (1975) could be replicated with the present stimulus display. Second, we wanted to investigate whether small surround-induced differences in the color of the stationary test field could influence CMAEs. Third, we wanted to investigate whether, in the absence of the colored stationary surrounds used during adaptation, addition of the same colors to the stationary test disk (which had rotated during adaptation) could induce appropriate CMAEs.

\section{Method}

During adaptation, a yellow (wavelength-constant) dotted disk changed its direction of rotation, simultaneously with the alternation of the color (red or green) of the stationary dotted surround (see Adaptation, Figure 1). A narrow dark annulus separated the disk and surround. It subtended $0.5^{\circ}$ of visual angle in width.

The stimulus conditions were counterbalanced between observers to protect against observer bias and stimulus artifacts. Thus, half the observers saw the clockwise $(\mathrm{CW})$ rotating disk paired with the red surround and the counterclockwise (CCW) rotating disk paired with the green surround; the other half saw the $\mathrm{CW}$ rotating disk paired with the green surround and the CCW rotating disk paired with the red surround. Since no observer bias or stimulus artifacts

Figure 1. The adapting and test conditions of Experiment 1. Subjects adapted to a clockwise $(\mathrm{CW})$ rotating yellow $(\mathrm{V})$ disk paired with a stationary red (R) surround, alternated with a counterclockwise (CCW) rotating $Y$ disk paired with a stationary green $(G)$ surround (see Adaptation). Both the disk and the surround were patterned with randomly placed dots. During testing (see Test), the observers reported whether the stationary disk and/or surround appeared to rotate or not. In the first three tests (rows 1-3), the surrounds were present with the disks; in the fourth and fifth tests (rows 4-5), they were absent. The direction of perceived motion (seen by all observers) and the average duration of that perceived motion are given below each test pattern. The single and double stars indicate whether the mean duration of the contingent motion aftereffects differed significantly from zero at the 0.05 or 0.01 level (Mann-Whitney $U$ statistic), respectively.
ADAPTATION
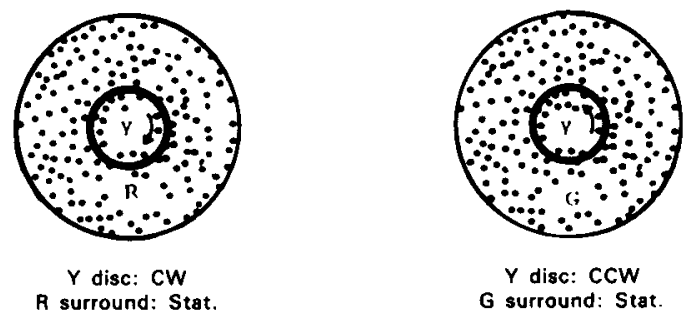

TEST
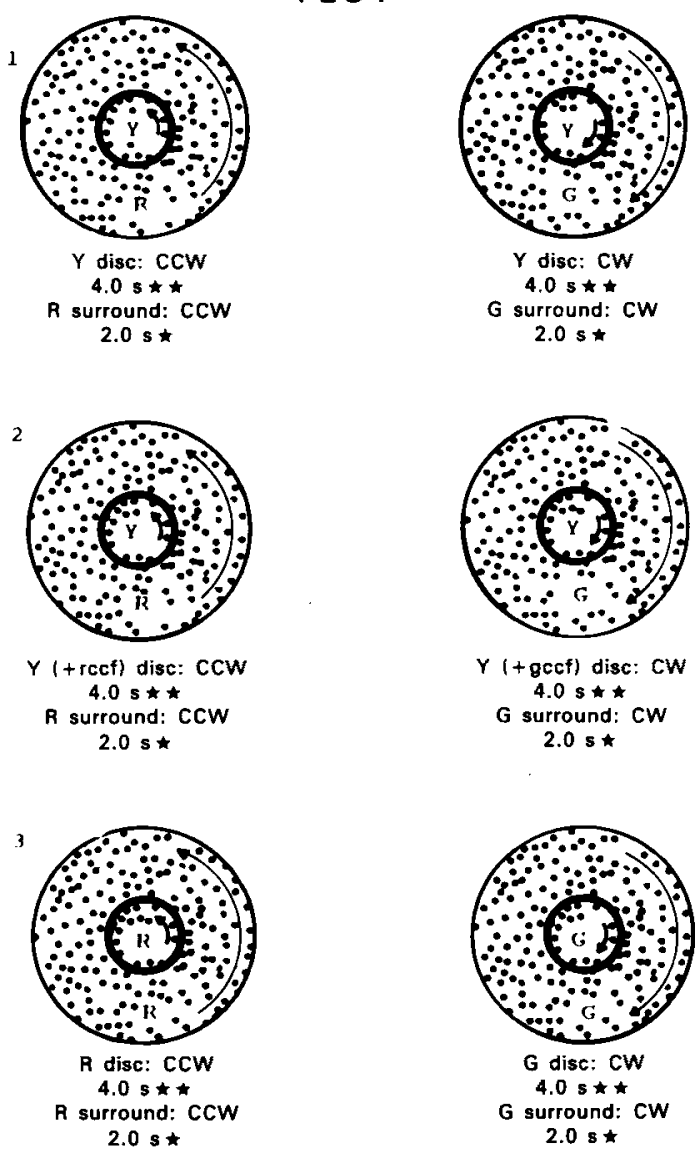

4

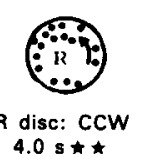

5

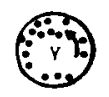

$Y(+\mathrm{rccf})$ disc: $\mathrm{CCW}$ $4.0 \mathrm{~s} \star \star$

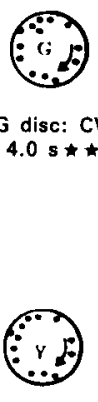

G disc: $\mathrm{CW}$ $4.0 \mathrm{~s} \star$

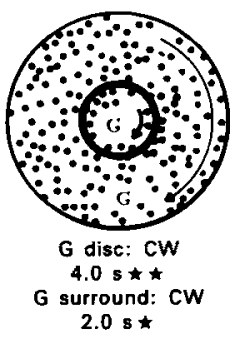

$Y(+$ gccf) disc: $\mathrm{CW}$ $4.0 \mathrm{~s} \star \star$ 
were encountered, we present the results below (and in the other experiments as well) as if all observers adapted to the same conditions.

The test conditions are given in Figure 1. The observers were first shown the stationary adapting patterns (Test, row 1), then variations of it-for example, red and green disks replacing the yellow disk (Test, row 3). During all test conditions, the observers were required to fixate the center of the stimulus display, just as they had done during adaptation.

\section{Results and Discussion}

The results from both the experienced and naive observers are summarized in Figure 1. After adapting to the $\mathrm{CW}$ rotating yellow disk paired with a red surround (which made the disk appear slightly greenish yellow), all observers reported (see the first row under Test) that the stationary disk-surround pattern appeared to rotate $\mathrm{CCW}$ (i.e., in the direction opposite to adaptation). And, after adapting to the $\mathrm{CCW}$ rotating yellow disk paired with a green surround (which made it appear slightly reddish yellow), all observers reported that the stationary pattern appeared to rotate $\mathrm{CW}$. Perceived motion was seen in both the central disk and the surround, but it was judged by all the observers to be stronger in the former, insofar as it persisted longer.

In the second test condition, we aimed to discover whether the surround color effect on the disk would still operate when the perceived color of the disk was held constant for both the red and the green surround by adding color-correcting filters to the yellow light illuminating the disk. When this was done (Test, row 2), the disk appeared a constant yellow-in spite of changes to the surround color-but the direction of the perceived CMAEs remained the same, and their magnitude was undiminished (i.e., their persistence was not shortened).

When both the disk and the surround were made the same color-either red or green (Test, row 3)-or when the disk alone (with the surround removed) was made red or green (Test, row 4)-the direction of the CMAEs did not change. That is, when the disk plus surround, or the disk alone, were red, the CMAEs appeared $\mathrm{CCW}$, and when they were green, the CMAEs appeared $\mathrm{CW}$. This was true even when the yellow disk was presented without the colored surrounds, but with either the red or the green color-correcting filter (Test, row 5). This established that a wavelength difference of about $3 \mathrm{~nm}$ (586 as opposed to $589 \mathrm{~nm}$ ) suffices to elicit color CMAEs.

These results therefore confirm and extend those of Potts and Harris (1975), by showing that MAEs from a constantwavelength rotating disk can be made contingent on the color of a stationary surround (Test, row 1), even when the apparent change of color induced by the surround is removed (Test, row 2) or opposed (Test, row 3 ). During testing, the surround itself is not necessary for obtaining CMAEs: When the disk is weakly (Test, row 5) or strongly (Test, row 4) tinged with the color of the absent surround, CMAEs appropriate in direction to the surround color can still be obtained. The same pattern of results was found in another experiment (not reported here), in which the color-correcting filters were added to the light illuminat- ing the yellow disk during adaptation so that it always appeared to be the same yellow, despite changes in the surround color.

The data from this experiment suggests that color information in one area of the visual field can become associated with motion information in another area to give CMAEs. During testing, color will trigger CMAEs from a stationary test field, whether it is presented in the area adapted to motion, or whether it has been held constant in wavelength or appearance during adaptation.

Although vivid color CMAEs could be elicited by the stationary adaptation patterns, our attempts to elicit motioncontingent color aftereffects (Hepler, 1968), with achromatic versions of the moving (dotted) adaptation patterns, were unsuccessful. The failure may lie in the nature of the adaptation and test patterns: Hepler, using upward and downward moving bars, instead of $\mathrm{CW}$ and $\mathrm{CCW}$ rotating dotted disks, was able to elicit motion-contingent color aftereffects.

\section{EXPERIMENT 2}

In Experiment 1, the surround was patterned with the same dots as the disk was, and the width of the dark annulus between the disk and the surround was narrow, subtending $0.5^{\circ}$ of visual angle. In Experiment 2, we explored the effects of increasing the width of the annulus (to about $5^{\circ}$ ), and of removing the dots from the surround. These two manipulations enabled us to reduce the possible effects of stray and scattered light upon the build-up of CMAEs during adaptation, to test whether color can become associated with movement separated by a large visual subtense, and to determine whether the similarity between the spatial properties of the disk and of the surround is necessary for such an association.

\section{Method}

In this experiment, the disk was dotted and it was illuminated with yellow light. The surrounds were unpatterned, and they were changed from red to green, and vice versa, as the direction of rotation of the disk reversed. The inner diameter of the dark annulus was $9.5^{\circ}$ of visual angle $(20 \mathrm{~cm}$ ), and its outer diameter (and so the inside diameter of the surround) was $14.3^{\circ}$ of visual angle $(30 \mathrm{~cm})$, giving an annulus width of $4.8^{\circ}(10 \mathrm{~cm})$. Under such conditions, the effects of simultaneous color contrast were greatly weakened.

\section{Results and Discussion}

In the first test condition (see Figure 2, Test, row 1), the subjects viewed stationary versions of the adapting displays and reported color CMAEs from the disk. Thus, after adapting to the yellow disk rotating $\mathrm{CW}$, plus green surround, alternating with yellow disk rotating $\mathrm{CCW}$, plus red surround (see Adaptation conditions), the stationary yellow disk appeared to rotate $\mathrm{CW}$ when the surround was red, and CCW when the surround was green. These effects were consistent across the 4 experienced observers, though their measured durations were short, with a mean of about $1 \mathrm{sec}$ (the durations are given to the nearest half second in this and all subsequent figures). When the sur- 
round and the disk were illuminated with the same color (Figure 2, Test, row 2), CMAEs became stronger (their duration rose to about $2.5 \mathrm{sec}$ ), and their direction was still appropriate to the surround color, even though the color of the disk had changed from the yellow used during adaptation. When the surrounds were made white, and the disk red or green (Figure 2, Test, row 3), CMAEs from the disk, though weaker than those with colored surrounds, were still consistently appropriate to the surround color, even though the surround color did not change. When the disk was made red, and the surround green (or vice versa; Figure 2, Test, row 4), CMAEs were very weak. Their reported direction of motion, when the subjects were forced to choose, was appropriate to the currently visible surround color, but their durations were less than $1 \mathrm{sec}$. A similar pattern of results was found in an otherwise identical experiment (not reported here) in which the annulus width was $3^{\circ}$, rather than $4.8^{\circ}$, and in which all 4 experienced observers and the 8 naive subjects participated.

The results of Experiment 2 confirm the general conclusion of Experiment 1; namely, surround color can become associated with disk motion. Moreover, the association can be built up across a gap of $4.8^{\circ}$, and it does not depend on whether or not the disk and the surround have the same spatial properties (i.e., the surround can be homogeneous or randomly dotted like the disk). As in Experiment 1, CMAEs can be elicited from the disk by illuminating it with the colors used in the surround during adaptation, even when the color of the surround is held constant during testing.

\section{EXPERIMENT 3}

The results from Experiments 1 and 2 show that, in the visual system, color information arising from a stationary surround can become associated with motion information arising from a central disk, even when the linkage must occur across a wide annulus. The purpose of Experiment 3 was to investigate whether color information can transfer in the opposite direction, from a stationary inner disk to a moving outer surround.

\section{Method}

The disk was unpatterned, and it was illuminated with red or green light. The surround was patterned, and it was always illuminated with yellow light, which was color corrected to compensate for any

Figure 2. The adapting and test conditions of Experiment 2. Subjects adapted to a counterclockwise (CCW) rotating yellow (Y) disk paired with a stationary red $(R)$ surround alternated with a clockwise (CW) rotating $Y$ disk paired with a stationary green (G) surround. The disk was patterned with randomly placed dots; the surround was unpatterned. The color changes in the appearance of the inner disk during adaptation were compensated for by adding colorcorrecting filters to the light beams illuminating the disk. The disk and surround were separated by a dark annulus $4.8^{\circ}$ in width. For other details, see caption to Figure 1.

ADAPTATION
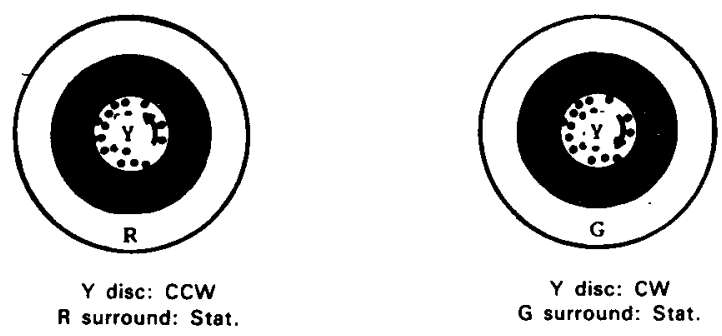

TEST

1

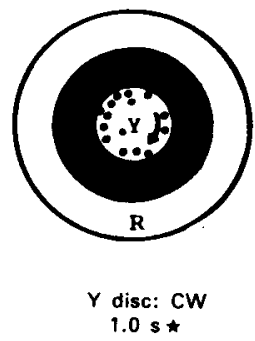

2

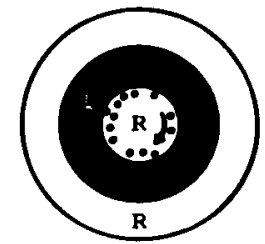

disc: $\mathrm{CW}$

$2.5 s \star \star$
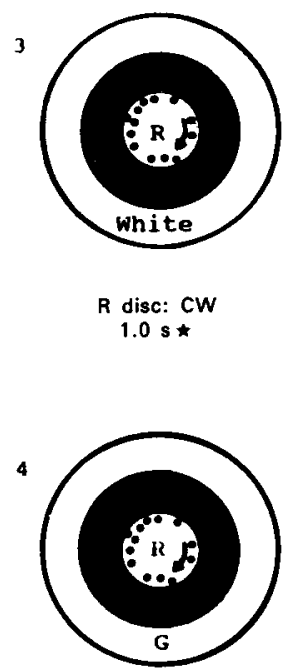

R disc: CW

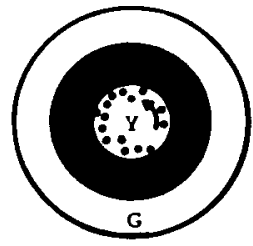

$Y$ dise: $\mathrm{CCW}$

$1.0 \mathrm{~s}$

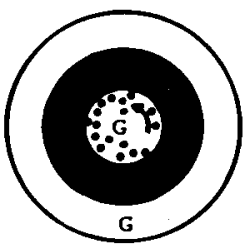

G disc: $\mathrm{CCW}$ 2.5 s $\star$
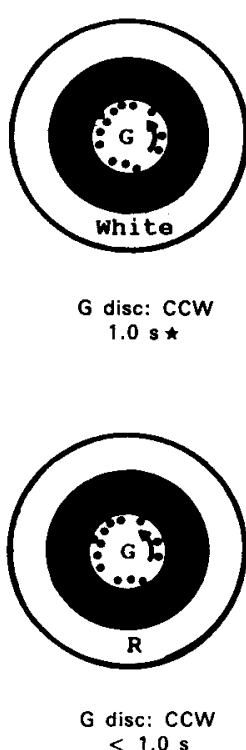
simultaneous color contrast from the disk. Its direction of rotation was reversed at the same time as the color of the disk changed from red to green and vice versa. The annulus was the same as had been used in Experiment 2, so that the gap between the disk and the surround was $4.8^{\circ}$.

\section{Results and Discussion}

When tested on stationary versions of the adapting fields (see Figure 3, Test, row 1), the 4 experienced observers reported CMAEs from the surrounds, which were appropriate to the color of the disk during adaptation. Thus after adapting to $\mathrm{CW}$ motion of the yellow surround, paired with a red disk, alternating with $\mathrm{CCW}$ surround motion, paired with a green disk, the subjects reported $\mathrm{CCW}$ CMAEs from the yellow surround when the disk was red, and CW CMAEs when it was green. These CMAEs were vivid and long-lasting; their duration averaged about $4.5 \mathrm{sec}$. When the disk was made yellow, and the patterned surround was red alternating with green (Figure 3, Test, row 2), the CMAEs were still appropriate to the disk color during adaptation, even though the disk was wavelengthconstant during testing. However, these CMAEs were weaker than those obtained in the first test, and their mean duration was about $1 \mathrm{sec}$. When the disk was illuminated with red (or green) light at the same time as the surround (Figure 3, Test, row 3), mean CMAE duration increased to about $2 \mathrm{sec}$. It rose still further $(2.8 \mathrm{sec})$ when the inner diameter of the dotted surround was expanded to include the area of the annulus (i.e., the dark, $4.8^{\circ}$ wide annulus was made the same texture and color as the surround during testing; see Figure 3, Test, row 4).

This experiment shows clearly that the lateral link between color and motion information is not unidirectional. It does not depend on surrounding the moving stimulus with color. Rather, the transfer can operate in the opposite direction-from the disk to the surround-across a $4.8^{\circ}$ gap. As with transfer from surround to disk, color added to the motion-adapted retinal area can trigger CMAEs appropriate to the colors presented to a different retinal area during adaptation.

\section{EXPERIMENT 4}

Experiment 3 showed that color information from mechanisms stimulated by the disk can become associated with motion information from mechanisms stimulated by the motion of the surround. In Experiment 4, we asked whether motion information can become linked in a similar way.

\section{Method}

The adaptation conditions were identical to those of Experiment 2. During adaptation, the disk was always patterned and yellow (colorcorrected to remain wavelength-constant). The surround was unpatterned, and it changed between green and red at the same time as the disk reversed its direction of motion. The annulus was $4.8^{\circ}$ of visual angle in width.

\section{Results and Discussion}

When tested with stationary versions of the adapting displays (Figure 4, Test, row 1), the observers reported

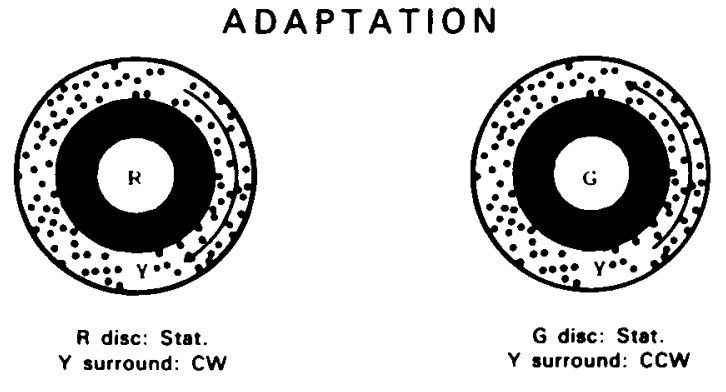

TEST
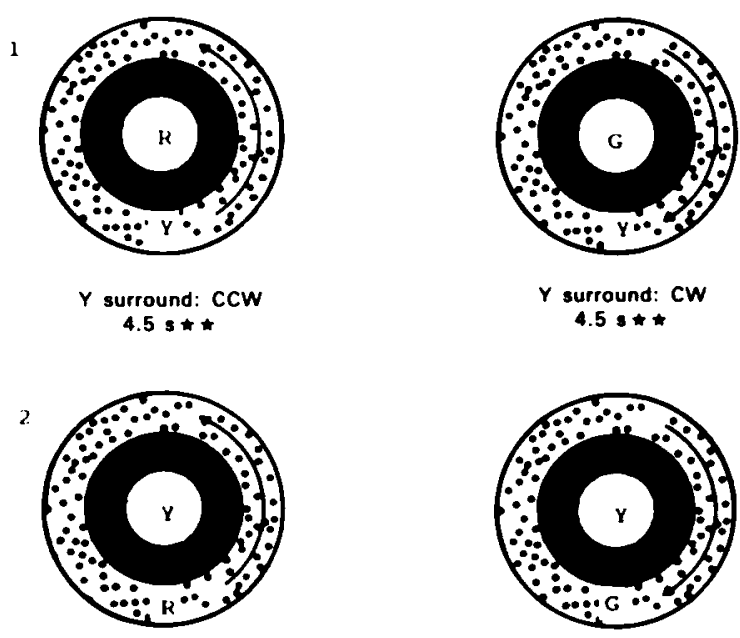

A surround: CCW

G surround: $C W$ $1.0 \mathrm{~s} *$
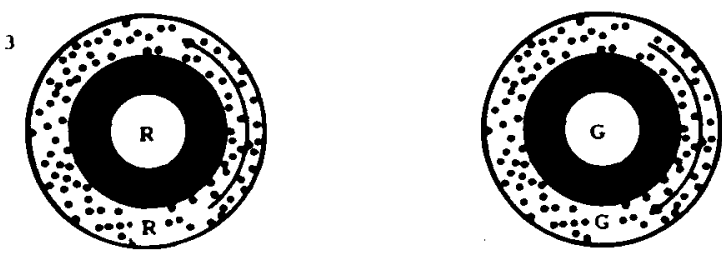

R surround: $\mathrm{CCW}$ $2.0 \mathrm{~s}$ *

$G$ surround: $\mathrm{CW}$ $2.0 * \star$
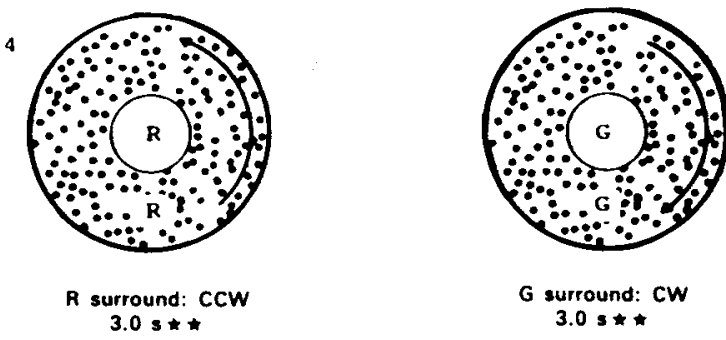

Figure 3. The adapting and test conditions of Experiment 3. Subjects adapted to a stationary red $(R)$ disk paired with a clockwise (CW) rotating yellow (Y) surround alternated with a stationary green (G) disk paired with a counterclockwise (CCW) rotating $Y$ surround. The disk was unpatterned; the surround was patterned with randomly placed dots. For other details, see the caption to Figure 1. 


\section{ADAPTATION}
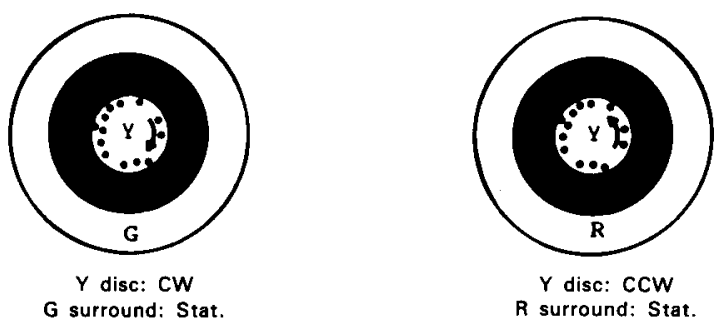

\section{TEST}
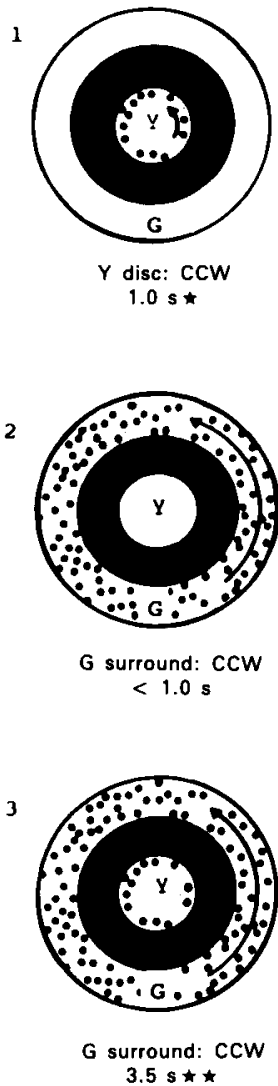

Figure 4. The adapting and test conditions of Experiment 4. Subjects adapted to a clockwise (CW) rotating yellow (Y) disk paired with a stationary green (G) surround alternated with a counterclockwise (CCW) rotating $Y$ disk paired with a red $(R)$ surround. The disk was patterned with randomly placed dots; the surround was unpatterned. For other details, see the caption to Figure 1.

CMAEs on the dotted yellow disk that were appropriate to the surround color (i.e., opposite to the direction of motion of the disk during adaptation). The CMAEs had a mean duration of about $1 \mathrm{sec}$ for the 4 experienced observers. When the surround was patterned with dots (and alternately red and green) and the disk unpatterned (but always yellow), CMAEs were obtained from the surround that were appropriate to its color (Figure 4, Test, row 2), though they were judged to be weaker than those of the previous test condition. When the conditions were the same as in row 2, but with dots added to the disk (Test, row 3), CMAEs were elicited by both the disk and the surround and in the same direction for both. However, the CMAEs elicited by the surround were of longer duration (about $3.5 \mathrm{sec}$ ).

This experiment confirms that color and motion can be linked across widely separated retinal positions in producing CMAEs. That is, motion information arising from the mechanisms responding to rotating disks can be linked to color information arising from the mechanisms responding to colored stationary surrounds.

\section{EXPERIMENT 5}

The previous experiments demonstrate that links between color and motion information can be produced between parts of the visual field that are separated by a wide $\left(4.8^{\circ}\right)$ dark annulus. They do not, however, reveal what effects, if any, are occurring in the annulus itself. This can be determined by presenting colored test stimuli to the retinal area that was stimulated during adaptation only by the dark annulus. Can CMAEs be found in a retinal area unstimulated by either color or motion during adaptation?

\section{Method}

During adaptation, both the disk and the surround were patterned, and they rotated in opposite directions. However, the disk and surround were always of different colors. Thus when the disk rotated $\mathrm{CCW}$ and the surround $\mathrm{CW}$, they were always green and red, respectively. When their directions of motion reversed, their colors also changed. The annulus subtended $4.8^{\circ}$ of visual angle in width.

\section{Results and Discussion}

Vivid CMAEs were perceived on stationary versions of the adapting fields by the 4 experienced subjects (see Figure 5, Test, row 1). On the disks, their mean durations were over $5 \mathrm{sec}$, and on the surrounds about $2 \mathrm{sec}$. The disk and surround were then made black, and the annulus filled with a black and white dotted pattern like that used on the disk and surround during adaptation (Test, row 2). When this test pattern was illuminated with red light, the annulus appeared to move $\mathrm{CCW}$, and when with green light, it appeared to move $\mathrm{CW}$. These effects had a mean duration of about $4 \mathrm{sec}$. If the dotted disk or the patterned surround were presented alone, or in combination with the patterned annulus (not shown), CMAEs were always observed. If the test field included the disk, mean durations were about $4 \mathrm{sec}$, and if it included the surround, they were about $2 \mathrm{sec}$.

These results are consistent with the idea that the visual mechanisms that respond to the annulus, but are not stimulated directly during adaptation, can nevertheless yield CMAEs, if information about color and motion have been transferred between two neighboring sets of mechanisms and passed through their domain. However, it cannot be ruled out that fixation was imperfect during adaptation 


\section{ADAPTATION}

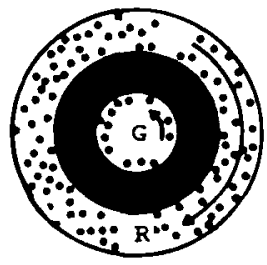

G disc: $\mathrm{CCW}$ R surround: $\mathrm{CW}$

\section{TEST}

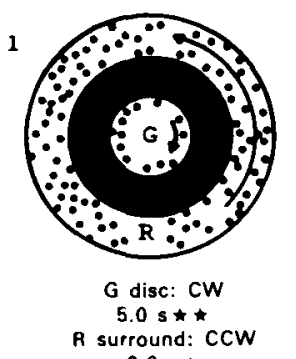

$2.0 \mathrm{~s} *$
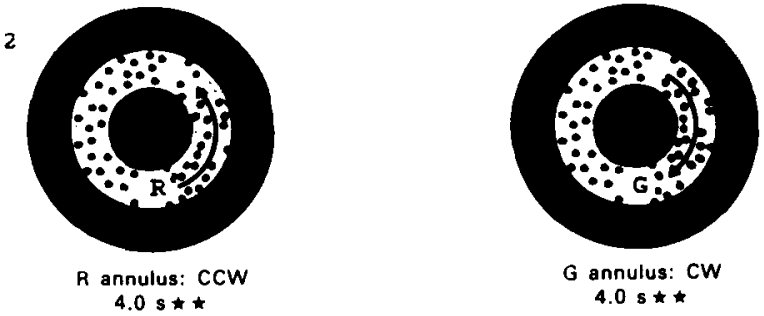

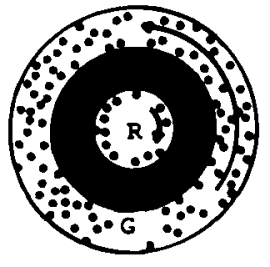

A disc: $\mathrm{CW}$ $G$ surround: $C C W$
$(7.0 \mathrm{sec})$ as those obtained from the disk or surround alone (see Figure 6, Test, row 1). If scattered light within the eye was contributing importantly to the CMAEs elicited by the annulus, a reduction in the strength of the CMAEs would be expected when the dimensions of the annulus were narrowed and the distance from the motion and color contours made greater.

\section{EXPERIMENT 6}

In the previous experiment, color and direction of motion were always paired in the same way on the disk and on the surround. So, if the pattern, whether on the disk or on the surround, was green, it always moved $\mathrm{CCW}$, and if it was red, CW. Thus, whatever the color of the patterned annulus, the effect on the visual mechanisms responding to it arising from those responding to the disk

\section{ADAPTATION}
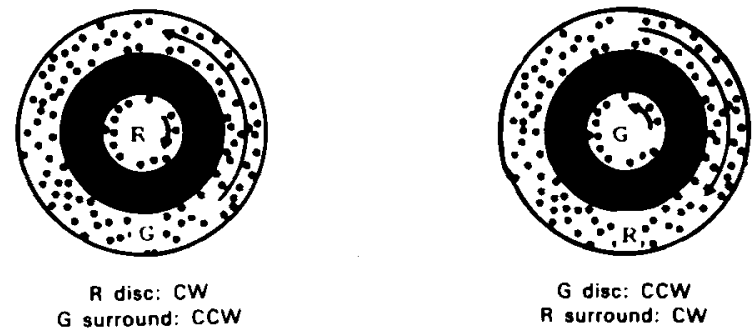

G surround: $\mathrm{CCW}$

\section{TEST}
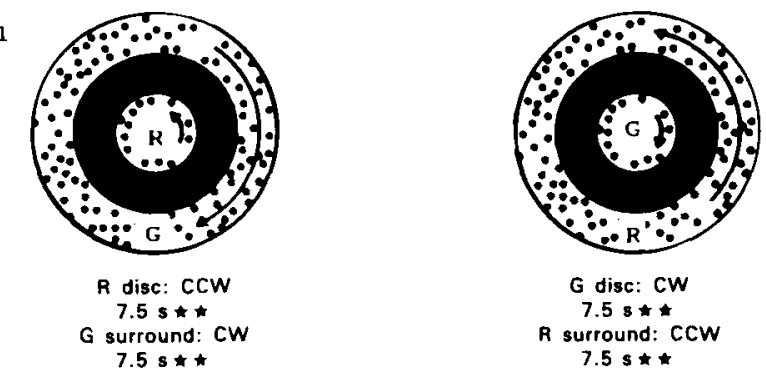

G disc: $\mathrm{CW}$

$7.5 \mathrm{~s} \star$

R surround: $\mathrm{CCW}$

$7.5 \mathrm{~s} \star$
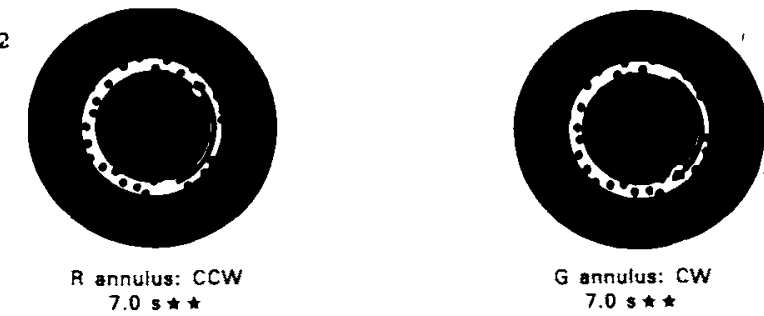

Figure 6. A test for the effects of scattered light upon the buildup of color-contingent motion aftereffects in retinal areas not stimulated by color or motion during adaptation. Subjects adapted to a clockwise $(\mathrm{CW})$ rotating red $(\mathrm{R})$ disk paired with a counterclockwise $(\mathrm{CCW}$ ) rotating green $(\mathrm{G})$ surround alternated with a $\mathrm{CCW}$ rotating $G$ disk paired with a $C W$ rotating $R$ surround. Both the disk and the surround were patterned with randomly placed dots. For other details, see the caption to Figure 1. we still obtained strong color CMAEs in the narrow annulus region. Furthermore, their duration was as long 
and the surround would be to generate CMAEs in the same direction. In Experiment 6, the direction of these two possible influences on the mechanisms responding to the annulus were put into conflict, so that the possible dominance of one over the other could be assessed.

\section{Method}

During adaptation, both the disk and the surround were patterned and they were illuminated with the same color. However, their directions of rotation were opposite. Thus when the disk and the surround were red, the disk rotated $\mathrm{CCW}$ and the surround $\mathrm{CW}$, and when they were green, the disk rotated $\mathrm{CW}$ and the surround $\mathrm{CCW}$. The annulus subtended $4.8^{\circ}$ of visual angle in width.

\section{Results and Discussion}

When the stationary versions of the adapting fields were presented (see Figure 7, Test, row 1), CMAEs were obtained from both the disk and the surround. As might be expected, since this is a standard split-field CMAE adaptation paradigm, these CMAEs were vivid. For our $4 \mathrm{ex}$ perienced subjects, the mean durations of the CMAEs elicited by the disk were over $5 \mathrm{sec}$, and those elicited by the surround, over $2 \mathrm{sec}$. Furthermore, tests were then made to determine if any CMAEs could be elicited by the annulus. First, the patterned disk was replaced with a dark disk, and the annulus was replaced with a black and white pattern like that of the surround (Figure 7, Test, row 2). When the surround and area occupied by the annulus during adaptation were illuminated with alternating red and green light, the entire pattern of dots appeared to move in the direction appropriate to surround adapting motion (mean duration $3 \mathrm{sec}$ ). When the test pattern was the same as in row 2 (Figure 7), except that the surround was made black and the disk made patterned (Figure 7, Test, row 3), the CMAEs (seen on the entire area of dots) were appropriate to disk adapting motion (mean duration $3.5 \mathrm{sec}$ ). When both the disk and surround were made black, and the area occupied by the annulus filled with the pattern, which was illuminated alternately with red and green light (Figure 7, Test, row 4), no consistent apparent motion was seen. There were hints of local motion at the outer and inner edges of the test pattern (which depended on fixation), but no global direction of motion was perceived.

Thus Experiment 6 suggests that the mechanisms responding to the retinal area occupied by the annulus are affected roughly equally by those responding to the disk and those responding to the surround. When the retinal area is stimulated by a pattern that includes the disk or the surround (but not both), the dots appear to move, as though captured by those in the disk or the surround. When tested in isolation with a dotted pattern, however, the annular region is not dominated either by more foveal or by more peripheral mechanisms, although local motion suggests that both are influencing the mechanisms responding to the annulus. A version of the experiment (not reported here), in which (during adaptation) both the disk and the surround moved in the same direction but had different colors that changed over when the direction of motion reversed, produced a similar pattern of results.
ADAPTATION

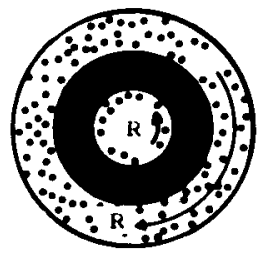

$R$ disc: $\mathrm{CCW}$

h surround: $\mathrm{CW}$

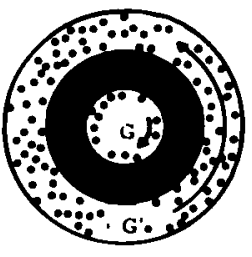

G disc: $\mathrm{CW}$ G surround: $\mathrm{CCW}$
TEST

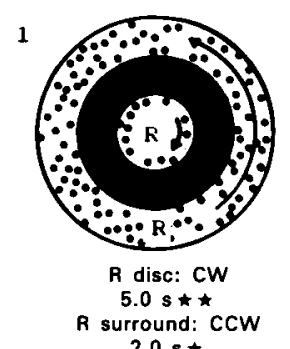

$2.0 \mathrm{~s} \star$

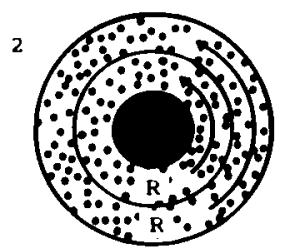

$R$ surround and $3.0 \mathrm{~s} \star \star$
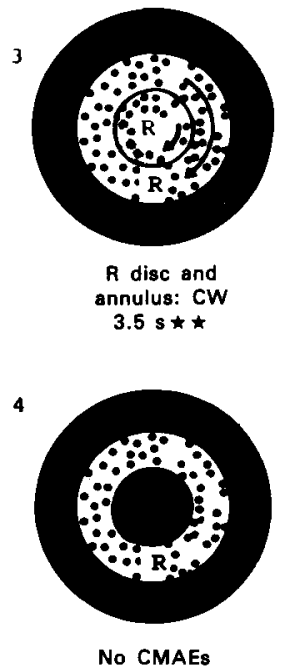
annulus: $\mathrm{CCW}$
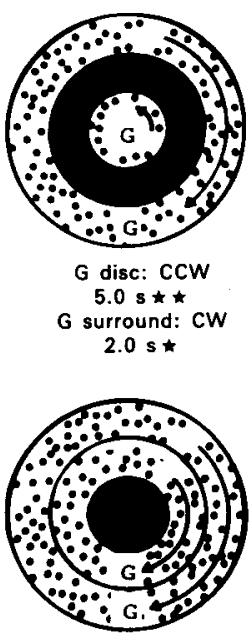

$G$ surround and annulus: $\mathrm{CW}$ $3.0 \mathrm{~s} \star \star$
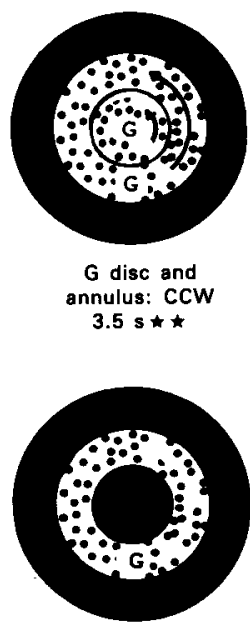

No CMAEs
Figure 7. The adapting and test conditions of Experiment 6. Subjects adapted to a clockwise (CW) rotating green (G) disk paired with a counterclockwise (CCW) rotating $G$ surround alternated with a $C C W$ rotating red $(R)$ disk paired with a $C W$ rotating $R$ surround. Both the disk and the surround were patterned with randomly placed dots. For other details, see the caption to Figure 1. 


\section{EXPERIMENT 7}

The foregoing experiments demonstrate that colorCMAEs can be elicited from retinal areas that are stimulated by only color or only motion or by neither color nor motion during adaptation, provided of course that some areas of the visual field are stimulated by color or by motion. This suggests that links between color and motion information arising from different regions of the visual field can occur, even when those regions are separated by a wide dark annulus. It could be argued, however, that such color CMAEs are more simply explained by large receptive fields, big enough to cover the disk, annulus, and surround. In fact, large receptive fields typically occur in the higher visual areas, like MT, and show motion sensitivity and possibly color responsivity as well (Albright, Desimone, \& Gross, 1984; Charles \& Logothethis, 1990). If so, there is no need to postulate lateral interactions to account for the effects from unstimulated retinal areas. Moreover, there is a remote possibility that scattering of light within the eye could, with our apparatus, transfer color and motion information to nominally unstimulated retinal areas. Thus, for example, the red light falling on a homogeneous disk might scatter within the eye to add a red tinge to a yellow rotating patterned surround, although we noticed no sign of such effects during adaptation. On this view, the lateral transfer of motion and color information would occur within the optics, not within the nervous system. Experiment 7 was designed for the purpose of testing these possibilities, first, by adapting the eye with colored, rotating disks and testing with (unpatterned) stationary, colored surrounds; and second, by adapting the eye with colored rotating surrounds and testing with (unpatterned) stationary, colored disks. Thus, each case represents an exchange between the color and texture features of the center and surround during the adaptation and test.

\section{Method}

In Part 1 , the subjects adapted to a red $\mathrm{CW}$ rotating disk alternated with a green $\mathrm{CW}$ rotating disk. The disk was patterned. The surround was always yellow and unpatterned. The hue of the surround did not appear to change as a result of simultaneous color contrast from the disk. In Part 2, the conditions were reversed: The subjects adapted to a red $\mathrm{CCW}$ rotating surround alternated with a green $\mathrm{CW}$ rotating surround. The surround was patterned. The disk was always yellow and unpatterned. In both Parts 1 and 2, the disk and surround were separated by a $4.8^{\circ}$ dark (unpatterned) annulus.

\section{Results and Discussion}

In Figure 8 are displayed the results obtained after adaptation to colored, rotating disks (Part 1). When tested on stationary versions of the adapting fields (Figure 8, Test, row 1), the 4 observers reported CMAEs from the disks, which were appropriate to (i.e., opposite) the color of the disk during adaptation. The CMAEs were vivid and very long-lasting ( $>10 \mathrm{sec}$ ). No CMAEs were observed in the surrounds. When the observers were tested on reversed versions of the adapting fields (Figure 8, Test, row 2)yellow, homogeneous disks paired with red- or green- colored, patterned surrounds-no CMAEs were reported in either the disks or the surrounds.

In Figure 9 are displayed the results obtained after adaptation to colored, rotating surrounds (Part 2). When tested on stationary versions of the adapting fields (Figure 9, Test, row 1), the 4 observers reported CMAEs from the surrounds that were appropriate to the color of the surround during adaptation. The CMAEs lasted more than $6 \mathrm{sec}$. No CMAEs were observed in the disks, either when they were presented with the surrounds (Figure 9, Test, row 1) or when they were alone (Test, row 2). When tested with red and green patterned disks paired with yellow patterned surrounds (Figure 9, Test, row 3) or paired with yellow homogeneous surrounds (the reverse versions of the adapting fields, not shown), the observers reported no CMAEs in either the disks or the surrounds. Further-

\section{ADAPTATION}
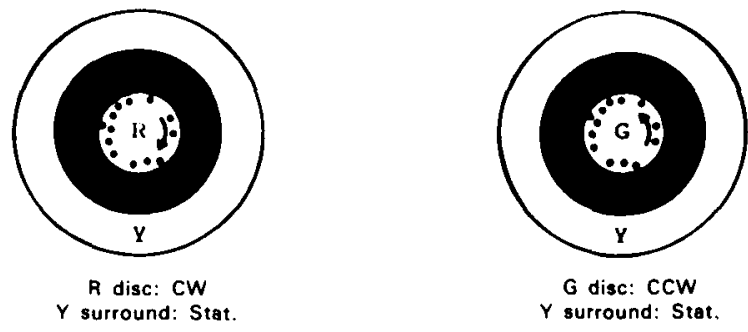

\section{TEST}
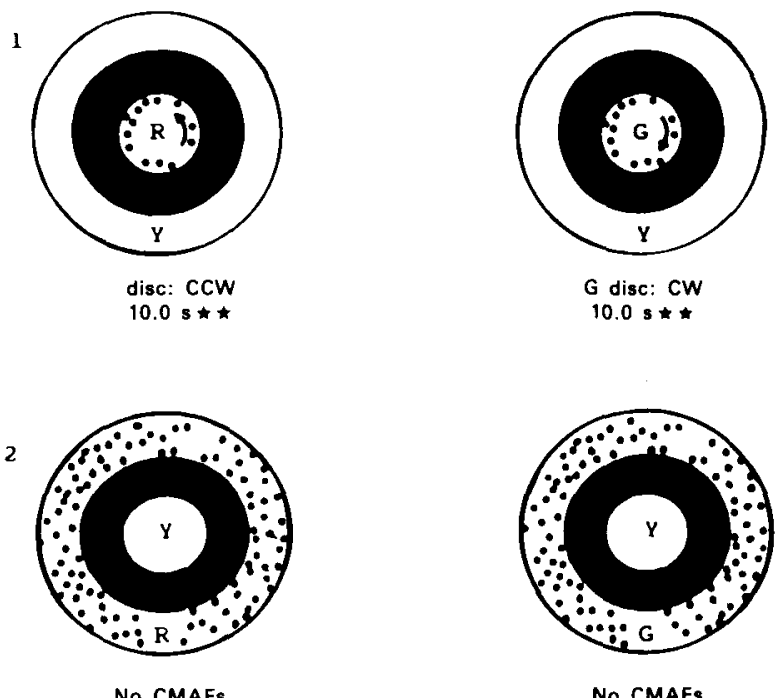

No CMAEs

Figure 8. The adapting and test conditions of the first part of Experiment 7 (Part 1). Subjects adapted to a counterclockwise (CCW) rotating green $(G)$ disk paired with a yellow $(Y)$ stationary surround alternated with a clockwise $(C W)$ rotating red $(R)$ disk paired with the same $Y$ stationary surround. During adaptation, the disk was patterned; the surround was homogeneous. They were separated by a dark annulus $4.8^{\circ}$ in width. For other details, see the caption to Figure 1. 
more, no CMAEs were reported in the disks when red and green patterned disks with red and green surrounds were presented-even though strong CMAEs were reported in the surrounds (Figure 9, Test, row 4).

Since no CMAEs were elicited from the unadapted retinal locations in any of the test conditions (provided that the observer fixated the center of the disk), we conclude that the lateral interaction effects reported above cannot be due to scattered light within the eye. Nor can they simply be due to large receptive fields, big enough to include the disk, annulus, and surround. However, as pointed out by one anonymous referee, we cannot completely rule out the latter possibility solely on the basis of these experiments. In the previous experiments (see Figures 3 and 4), color and motion were presented to disparate retinal areas during adaptation, and in the second test in each case, one feature was exchanged between center and surround during test (Figure 3, color; Figure 4, texture). These two cases show the smallest CMAE durations ( $1 \mathrm{sec}$ or less) of all tests other than those in Experiment 7 (in which both features were exchanged). In fact, the mean durations for Figures 3 and 4 (Test, row 2), do not differ significantly from those of the last experiments (Figures 8 and 9, Test, row 1). So it could be maintained that large receptive fields are responsible for the effects and that a similarity between test and adaptation configuration is also a contributing factor. However, this explanation does not offer an obvious reason for why CMAEs should be obtained from retinal areas stimulated during adaptation only by the dark annulus (Figure 6, Test, row 2). Here, the spatial configurations of the test and adapting stimuli were very different, yet vivid CMAEs could be obtained. Therefore, we think it more likely that CMAEs elicited from unstimulated retinal areas or from areas stimulated only with motion or color are due to lateral interactions within the visual system.

\section{GENERAL DISCUSSION}

The present experiments show that during adaptation to colored moving patterns, in which the color and motion are presented to different retinal areas separated by about $5.0^{\circ}$ of visual angle, both color and motion information are exchanged between the neural representations of the two stimulated areas of the visual field. They further show that the neural mechanisms responding to a retinal area between the two stimulated areas are affected by this linkage of information as well. Such associations most probably depend on lateral connections between quite widely separated neural mechanisms. The anatomical nature of such connections is obscure at present, but there is physiological evidence for their existence. Valberg et al. (1985), for instance, have shown that achromatic remote annular surrounds, with inner diameters of up to $50^{\circ}$ of visual angle, can influence the response of cells in the macaque's lateral geniculate nucleus to increments of luminance above that of a small $\left(4^{\circ} \times 5^{\circ}\right)$ achromatic adapting field. The annular surrounds shifted the intensity-response

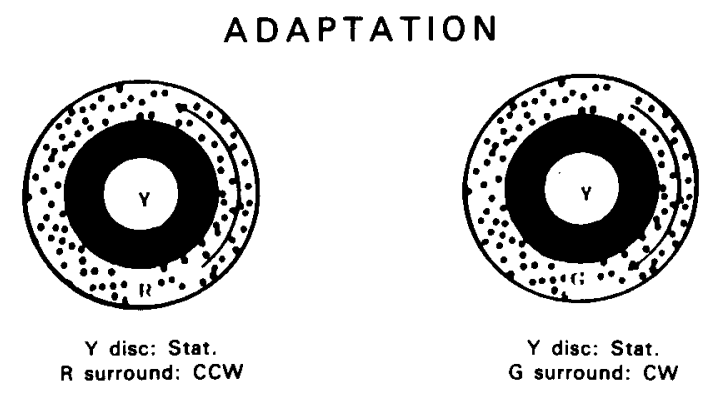

TEST

1
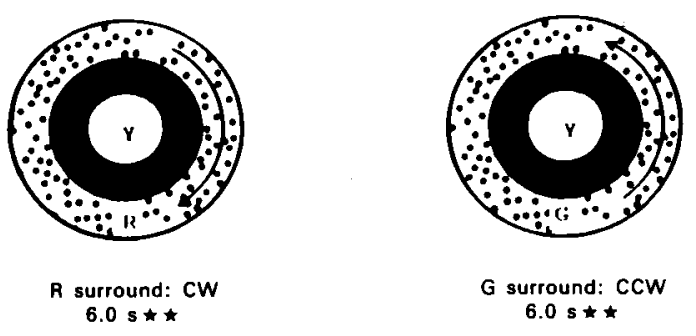

G surround: $\mathrm{CCW}$ $6.0 \mathrm{~s} \star \star$

2
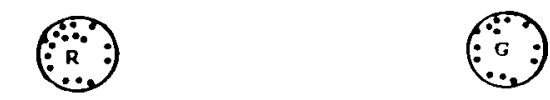

$R$ disc: No CMAEs

G disc: No CMAEs

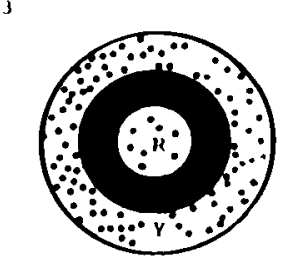

No CMAEs

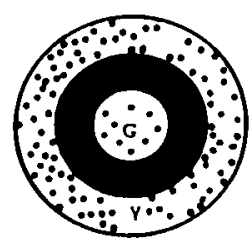

No CMAEs
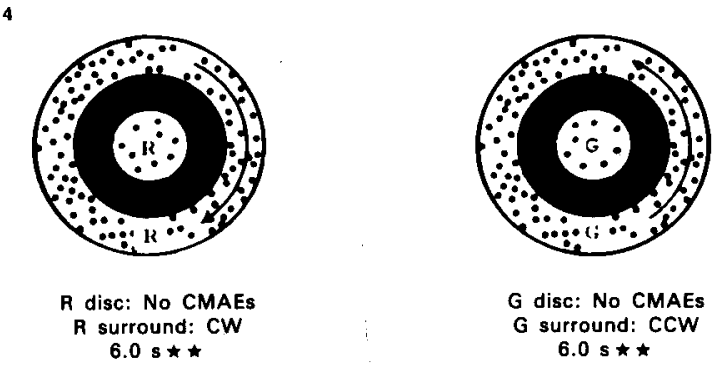

G disc: No CMAEs G surround: $\mathrm{CCW}$ $6.0 s \star \star$

Figure 9. The adapting and test conditions of the second part of Experiment 7 (Part 2). Subjects adapted to a counterclockwise $(C C W)$ rotating red $(R)$ surround paired with a yellow $(Y)$ stationary disk alternated with a clockwise (CW) rotating green (G) surround paired with the same $Y$ stationary disk. During adaptation, the disk was homogeneous; the surround was patterned. They were separated by a dark annulus $4.8^{\circ}$ in width. For other details, see the caption to Figure 1. 
curves to higher intensity ranges by up to $1 \log$ unit, without changing their shapes. In the context of adaptation experiments, it is interesting that adaptation to contrast produces just such a shift in the contrast response function of cat and monkey cortical neurons (Albrecht \& Hamilton, 1982). In other words, the "simultaneous contrast" effect of Valberg et al. (1985) from remote surrounds appears to produce similar functional changes in single neuron responses to the "successive contrast" effects within the classical receptive field found by Albrecht and Hamilton (1982), albeit in different species and at different anatomical sites.

Such remote surround effects would not directly explain our psychophysical results. To do so would require some additional assumptions. For example, one must suppose that surround effects can be chromatic, as well as achromatic, and thus differentially affect remote color-coding neurons. This could explain the exchange of color information between disks and surrounds in our experiments, and it would fit well with the suggestion of Valberg et al. (1985) that the light falling on large retinal regions is used to adjust the gain of single neurons within those regions and to optimize local discriminations of color and brightness. However, the exchange of motion information implied by our experiments, if it is to be explained in an analogous way, suggests that such a process is not confined to a level of visual analysis at which instantaneous or time-integrated averages of mean luminance and wavelength are represented. Rather, it must occur additionally (or instead), at a level of analysis at which primitives such as direction of motion are being (or have been) extracted. This line of reasoning also follows from the demonstrations by Potts and Harris (1975) that MAEs can be made contingent on surround orientation and spatial frequency as well as surround color. The functional role of such a mechanism would presumably be similar to that suggested by Valberg et al. (1985) for their unpatterned achromatic surround effects: to change aspects of the response of neurons to motion, in relation to motion elsewhere in the visual field. Thus the transfer of motion information suggests that some surround effects are not produced by a mechanism like a simple integrating photometer, an idea that is open to physiological investigation with displays like ours.

One apparently well-established fact about simple and contingent aftereffects is that they are confined to the adapted area of the retina (Gibson, 1933; Gibson \& Radner, 1937; Stromeyer, 1978). Our claims that CMAEs can be obtained from the retinal area stimulated during adaptation by an invariant dark annulus therefore appear to be somewhat heretical. Notice, however, that there is an important difference between our adapting procedure and those used in other studies. In previous work, a single retinal area has been adapted, or two adjoining areas have been simultaneously adapted by separate stimuli with similar properties but different values (for example, red patterns moving upward and green patterns moving downward). In our study, annulus effects were found when different retinal areas were adapted to stimuli whose properties and values were the same (e.g., red patterns rotating $\mathrm{CW}$ ). No CMAEs could be found from the retinal area adapted to the annulus, when the CMAEs from the disk and the surround were opposite in direction. They appeared only when the CMAEs from the surrounding retinal areas were in the same direction. Thus we believe that in the split-field adapting conditions, there is indeed some kind of contingent adaptation in the area of the visual field corresponding to the annulus, but that the effects from disk and surround cancel each other out, leaving no net annulus effect. This was probably also the case in the split-field adapting conditions of previous studies. When only one retinal region was adapted, there would be no contingent change in surround conditions, and so no spread of adaptation would be expected. Our Experiment 7 demonstrated such lack of spread, in a display whose spatial dimensions, though not the particular adapting conditions, were the same as those in the experiments demonstrating lateral transfer. We conclude, therefore, that the retinal area specificity of contingent adaptation is a myth. Adaptation can indeed spread to mechanisms responding directly to unstimulated regions of the visual field with appropriate adapting conditions.

There are two broad ideas about the neural origin of contingent aftereffects (for an overview, see Treisman, 1986). According to one view, originally proposed by McCollough (1965), contingent aftereffects reflect adaptation in doubleduty neurons, which respond (in the case of CMAEs) both to color and to motion. The other view (Mayhew \& Anstis, 1972; Murch, 1974, 1976) suggests that during contingent adaptation, links are built up between single-duty neurons, selective for color alone and for motion alone, perhaps by a process analogous to classical conditioning (Murch, 1974, 1976).

Our results can be accommodated by the first hypothesis. They suggest that double-duty neurons responding to one part of the visual field can be influenced by singleor double-duty units in another.

Our results, however, appear to create some problems for the second hypothesis. Conventional wisdom holds that the neural events mediating the experience of colored afterimages originate in the retina (Craik, 1940; Pöppel, 1986), whereas the motion aftereffect is believed to reflect the adaptation of local direction-selective neural mechanisms. In the rabbit, these occur in the retina (Barlow \& Hill, 1963), but in the cat (and thus probably in man, also), they are cortical (Hammond, Mouat, \& Smith, 1985, 1988; Vautin \& Berkley, 1977; von der Heydt, Hänny, \& Adorjani, 1978). This suggests that retina based or lateral geniculate based simultaneous color contrast effects must be combined in the cortex with motion information in order to generate color CMAEs. Indeed, good evidence supports this supposition. Color CMAEs do not transfer interocularly (Mayhew \& Anstis, 1972), but they can be dichoptically induced (Murch, 1974; Potts \& Harris, 1979). Murch (1974) explained his result by suggesting that during adaptation, monocular color units became 
linked to binocular motion units. This link could only be activated during testing if the eye that saw color during adaptation also saw it during testing.

Our results, however, would seem to indicate that, at least, surround-induced CMAEs do not result from twoway links between single-duty units representing different areas of the visual field. If this were so, then CMAEs should be obtained only when the stationary test field, and the colors, occupy the positions that they occupied during adaptation. Consider the case in which the disk was always patterned and yellow and reversed its direction of motion as the colors of the unpatterned stationary surround alternated. Color units responding to the surround would be linked to motion units responding to the disk. Since there was no movement in the surround and no change of wavelength in the disk, there is no obvious reason for single-duty color and motion units within those regions of the visual field to become linked to each other. When the stationary yellow disk is presented together with alternating colored surrounds, the links between surround color units and disk motion units will give CMAEs, and one would predict that alternating directions of rotation of the disk would give colored CMAEs on a white surround. But this scheme provides no mechanism for CMAEs from the stationary disk when it is made red or green and the surround is made achromatic (Experiment 2), or from the surround when it is patterned and red or green and the disk is unpatterned and yellow (Experiment 4), since no obvious link between color and motion within each separate region would be expected. Thus we conclude that, in its presently accepted form, the idea of links between single-duty units does not account for our data.

Our claims in this paper depend on comparisons between the occurrence and magnitude or duration of CMAEs within a particular experiment. There is, however, a tendency in the data (which does not affect our claims) that is puzzling and that may be interesting. The experiments are presented in the order in which they were performed. The data from Experiments 2-8 show a fairly consistent tendency for CMAE durations to increase in successive experiments, regardless of the particular adapting conditions, which were usually different both in their spatial arrangements and, for individual subjects, in the combination of adapting color and motion. Moreover, although some experiments were performed on the same day, there were gaps of days and weeks between others. This therefore does not seem to be merely an effect of cumulative adaptation, in the sense that repeated presentations of the same color and direction of motion were made to the same retinal areas. It might represent a gradual shift of criterion for reporting that CMAEs had ended, or some kind of generalized change in adaptability to this kind of stimuli. From the present data, we cannot speculate further about its origin, but this apparent change in CMAE duration might be worth investigation.

CMAEs of the type reported here cast no doubt on the view that the optimal stimuli for human motion-detecting units are defined by luminance rather than by color contrast. Although the backgrounds to our moving stimuli were of similar luminance, whatever their color, the moving elements (the dots) were always very different in luminance from the backgrounds. However, the strongest implication of such effects is that motion-sensitive units are also color-sensitive, and the weakest is that there are indeed strong links between the processing of color and of motion information early in human vision. It remains possible that color and motion are associated for one purpose (adjustment of the gains of individual channels) and segregated for another (analysis of different stimulus attributes). However, since the task of the human visual system, when presented with a colored moving object, is to tell the rest of the human brain that there is indeed one colored moving object, not one moving object and one colored object, the advantages of early intimacy over prolonged estrangement seem obvious.

\section{REFERENCES}

Albrecht, D. G., Hamilton, D. B. (1982). Striate cortex of monkey and cat: Contrast response function. Journal of Neurophysiology, 48, 217-237.

Albright, T. D., Desimone, R., \& Gross, C. G. (1984). Columnar organization of directionally selective cells in visual area MT of the macaque. Journal of Neurophysiology, 51, 16-31.

ANSTIS, S. (1975). What does visual perception tell us about visual coding? In C. Blakemore \& M. Gazzaniga (Eds.), Handbook of psychobiology (pp. 436-478). London: Academic Press.

Barlow, H. B., \& HiLl, R. M. (1963). Evidence for a physiological explanation of the waterfall phenomenon and figural after-effects. $\mathrm{Na}$ ture, 200, 1345-1346.

Carney, T., Shadlen, M., \& Switkes, E. (1987). Parallel processing of motion and color information. Nature, 328, 647-649.

Cavanagh, P., Tyler, C. W., Favreau, O. E. (1984). Perceived velocity of moving chromatic gratings. Journal of the Optical Society of America A, 1, 893-899.

Charles, E. R., \& Logothetis, N. (1990). MT responses to drifting low contrast homochromatic and heterochromatic gratings. Investigative Ophthalmology \& Visual Science, 31(Suppl.), 238.

CraIK, K. J. W. (1940). Origin of visual afterimages. Nature, 145, 512-513.

DeYoe, E. A., \& Van Essen, D. C. (1988). Concurrent processing streams in monkey visual cortex. Trends in Neural Science, 11, 219-226.

Favreau, O. E., Emerson, V. F., \& Corballis, M. C. (1972). Motion perception: A color contingent aftereffect. Science, 176, 78-79.

Gibson, J. J. (1933). Adaptation, aftereffect and contrast in the perception of curved lines. Journal of Experimental Psychology, 16, 1-31.

GiBSON, J. J., \& RADNER, M. (1937). Adaptation, aftereffect and contrast in the perception of tilted lines. Journal of Experimental Psychology, 20, 453-467.

Hammond, P., Mouat, G. S. V., \& SMith, A. T. (1985). Motion aftereffects in cat striate cortex elicited by moving gratings. Experimental Brain Research, 60, 411-416.

Hammond, P., Mouat, G. S. V., SMith, A. T. (1988). Neural correlates of motion aftereffects in cat striate cortical neurons: Monocular adaptation. Experimental Brain Research, 72, 1-20.

Hepler, N. (1968). Color: A motion-contingent aftereffect. Science, 162, 376-377.

MAYHEW, J. E. W., \& ANSTIS, S. M. (1972). Movement aftereffects contingent on color, intensity, and pattern. Perception \& Psychophysics, 12, 77-85.

McCollough, C. (1965). Color adaptation of edge-detectors in the human visual system. Science, 149, 1115-1116. 
MURCh, G. M. (1974). Color contingent motion aftereffects-multiple levels of processing? Vision Research, 14, 1181-1184.

Murch, G. M. (1976). Classical conditioning of the McCollough effect: Temporal parameters. Vision Research, 16, 615-619.

PöPPEL, E. (1986). Long-range color-generating interactions across the retina. Nature, 320, 523-525.

PotTs, M. J., \& Harris, J. P. (1975). Movement aftereffects contingent on the color of pattern of a stationary surround. Vision Research, 15, $1225-1230$.

Potts, M. J., \& Harrus, J. P. (1979). Dichoptic induction of movement aftereffects contingent on color and on orientation. Perception \& Psychophysics, 26, 25-31.

Ramachandran, V. S., \& Gregory, R. L. (1978). Does color provide an input to human motion perception? Nature, 275, 55-56.

SHAPLEY, R. (1990). Visual sensitivity and parallel retinocortical channels. Annual Review of Psychology, 41, 635-658.

Skowbo, D., Timney, B. N., Gentry, T. A., \& Morant, R. B. (1975). McCollough effects: Experimental findings and theoretical accounts. Psychological Bulletin, 82, 497-510.

STROMEYER, C. F., III. (1978). Form-color aftereffects in human vision. In R. Held, H. Leibowitz, \& H.-L. Teuber (Eds.), Handbook of sensory physiology: Vol. 8. Perception (pp. 97-142). Berlin: Springer.

Treisman, A. (1986). Properties, parts and objects. In K. R. Boff, L. Kaufman, \& J. P. Thomas (Eds.), Handbook of perception and human performance (pp. 1-70). New York: Wiley.

Troscianko, T. (1987). Perception of random-dot symmetry and apparent movement at and near isoluminance. Vision Research, 27, 547-554.
Troscianko, T., \& FAHLE, M. (1988). Why do isoluminant stimuli appear slower? Journal of the Optical Society of America A, 5, 871-880.

Valberg, A., Lee, B. B., Creutzfeldt, O. D., \& Tigwell, D. A. (1985). A simultaneous contrast effect of remote surrounds on intensityresponse curves of cells in the macaque LGN. Experimental Brain Research, 58, 604-608.

VAN ESSEN, D. C. (1986). Functional organization of primate visual cortex. In A. Peters \& E. G. Jones (Eds.), Cerebral cortex (Vol. 3, pp. 259-329). New York: Plenum.

VAN Essen, D. C., \& MaUnSell, J. H. R. (1983). Hierarchical organization and functional streams in the visual cortex. Trends in Neuroscience, 6, 370-375.

VAUtiN, R. G., \& BeRKLEY, M. A. (1977). Responses of single cells in cat visual cortex to prolonged stimulus movement: Neural correlates of visual aftereffects. Joumal of Neurophysiology, 40, 1051-1065.

von der Heydt, R., HÄNNY, P., \& Adorjani, C. (1978). Movement aftereffects in the visual cortex. Archives Italiennes de Biologie, 116, 248-254.

WOLFE, J. (1985). Fatigue and structural change: Two consequences of visual pattern adaptation. Investigative Ophthalmology \& Visual Science, 26(Suppl.), 84.

ZEKI, S. M. (1978). Uniformity and diversity of structure and function in the monkey prestriate visual cortex. Journal of Physiology, 277, 273-290.

(Manuscript received June 18, 1990; revision accepted for publication January 11, 1991.) 